\title{
Vibrational Coherence Spectroscopy Identifies Ultrafast Branching in an Iron(II) Sensitizer
}

F. Hainer ${ }^{1,2}$, N. Alagna ${ }^{1,2}$, A. Reddy Marri ${ }^{4}$, T. J. Penfold ${ }^{5}$, P.C. Gros ${ }^{4}$, S. Haacke ${ }^{* 3}$, T. Buckup*1,2

${ }^{1}$ Physikalisch Chemisches Institut, Ruprecht-Karls Universität, D-69120 Heidelberg, Germany

${ }^{2}$ Centre for Advanced Materials, Ruprecht-Karls Universität, D-69120 Heidelberg, Germany

3University of Strasbourg - CNRS, IPCMS, Strasbourg, France

${ }^{4}$ Université de Lorraine, CNRS, L2CM, F-54000 Nancy, France

${ }^{5}$ Chemistry-School of Nat. \& Env. Sci., Newcastle University, Newcastle Upon-Tyne, UK

${ }^{*}$ Corresponding Authors:

tiago.buckup@pci.uni-heidelberg.de, Phone: +49 (0)6221 54 8723, Fax: +49 (0)6221 548730

stefan.haacke@ipcms.unistra.fr, Phone: +33(0)388107171; Fax: +33(0)388107145 


\section{Contents}

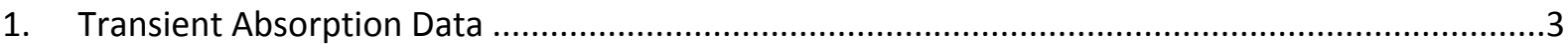

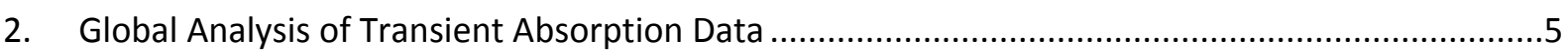

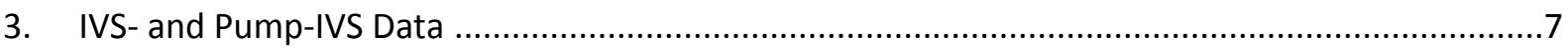

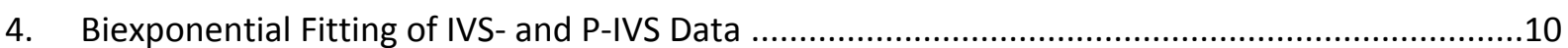

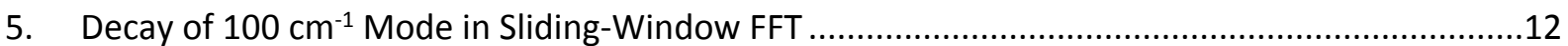

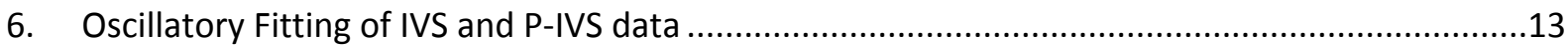

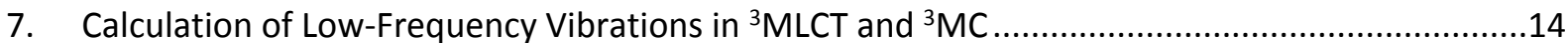




\section{Transient Absorption Data}

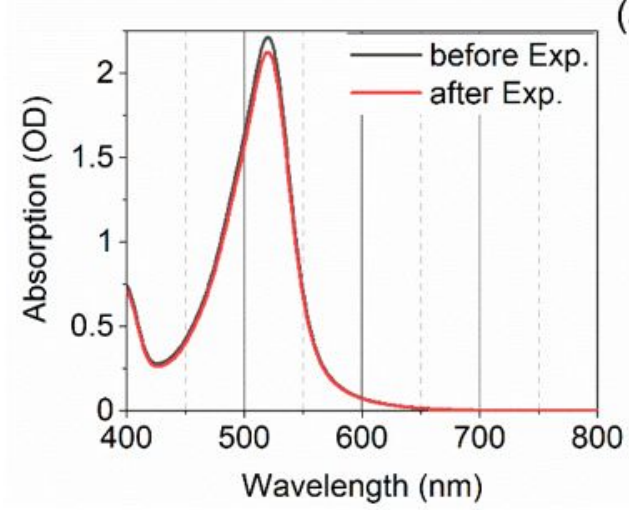

(a)

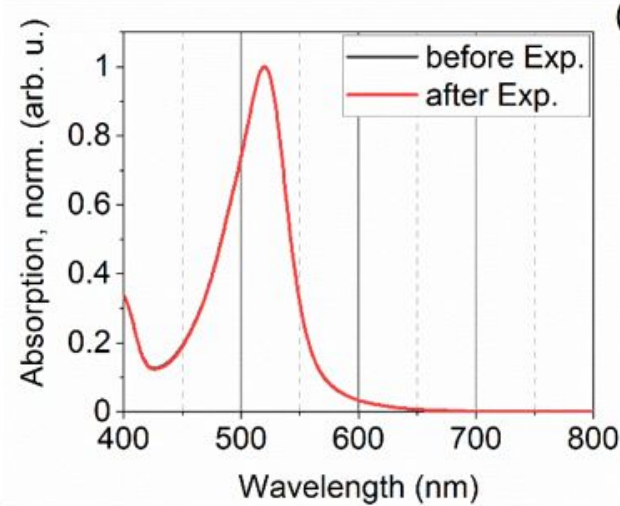

(b)

(c)

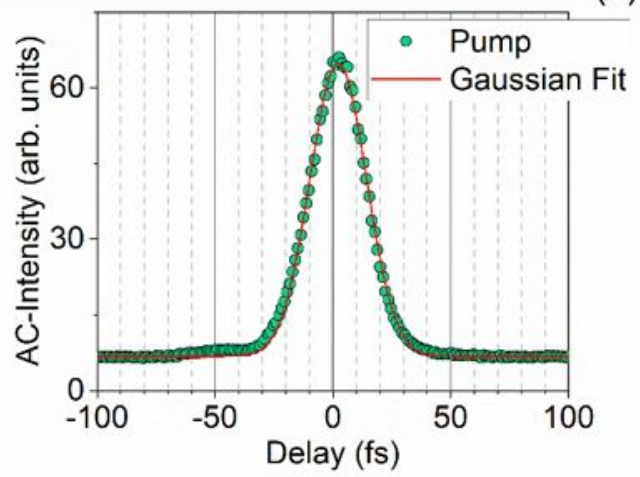

Figure S1: (a) Absolute and (b) normalized ground state absorption before and after TA measurements, recorded in a $2 \mathrm{~mm}$ cuvette. (c) Autocorrelation of pump pulse.

Absorption at $\lambda=520 \mathrm{~nm}$ decreases from 1.58 OD to 1.51 OD i.e. by $5 \%$. The comparison of the normalized absorption spectra (Figure S1b) shows no additional changes in the spectrum shape, indicating that the photo-damage does not result in any species absorbing in the observed spectral range.

The Gaussian fit of the autocorrelation of the pump pulse (Figure S1c) yields 28.3 fs FWHM corresponding to a $20.0 \mathrm{fs}$ pulse duration. 
(a)

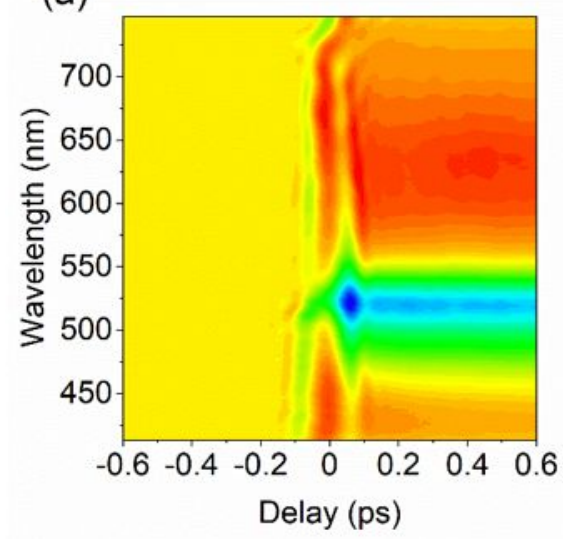

(c)

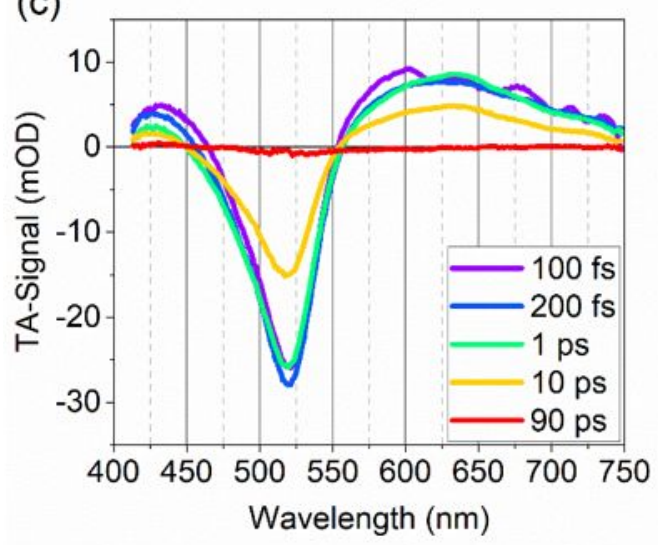

(b)
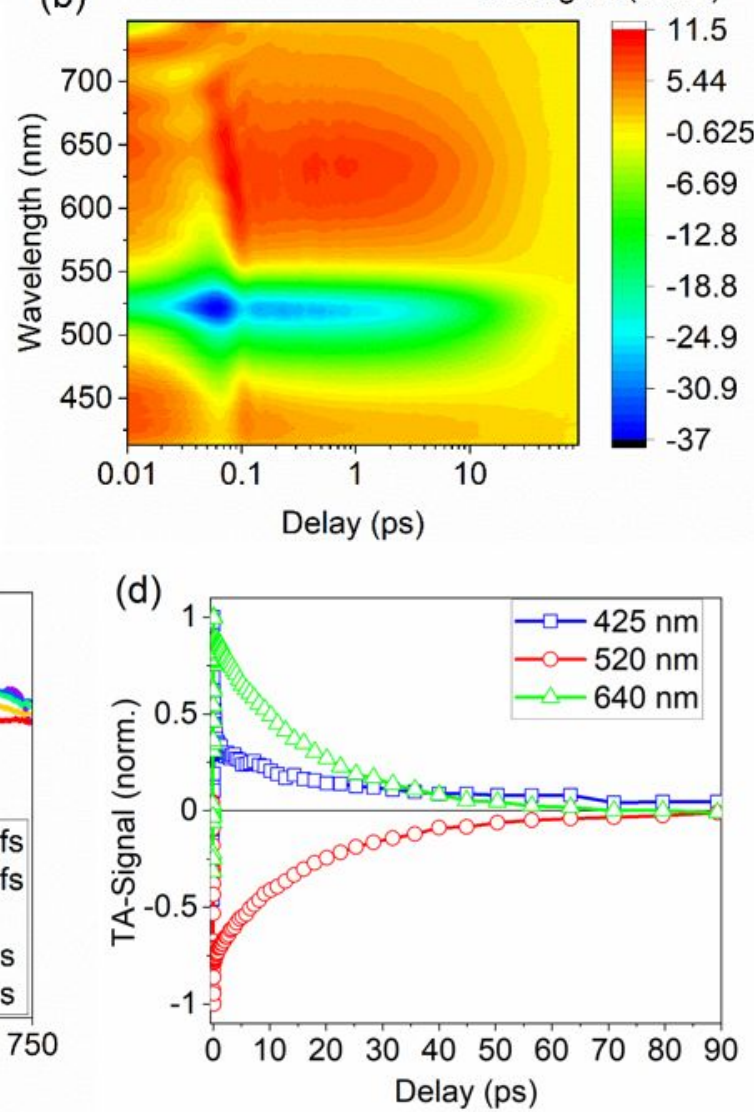

Figure S2: Transient absorption data. (a) Short time scale, (b) full data set in logarithmic timescale, (c) selected transient spectra and (d) normalized traces at selected wavelengths.

\section{Global Analysis of Transient Absorption Data}

Figure S3 shows the fitting with two and three exponential decays. The fit employing two decays fails to describe a blue shift of the broad excited state absorption band $(\lambda>575 \mathrm{~nm})$ overlapping with the ground state bleach around $\lambda=530 \mathrm{~nm}$ (see Figure S3 b). The fit employing three decays reproduces this blue shift and yields a featureless residual for delays larger than $500 \mathrm{fs}$ (Figure S2 d). The residual at delays below $500 \mathrm{fs}$ is due to signal modulations from the wavepacket dynamics investigated with IVS. The time constants obtained for the three decays are $220 \pm 6 \mathrm{fs}, 5.3 \pm 0.3 \mathrm{ps}, 19.9 \pm 0.2 \mathrm{ps}$ and their associated spectra are given in Figure S3f. 

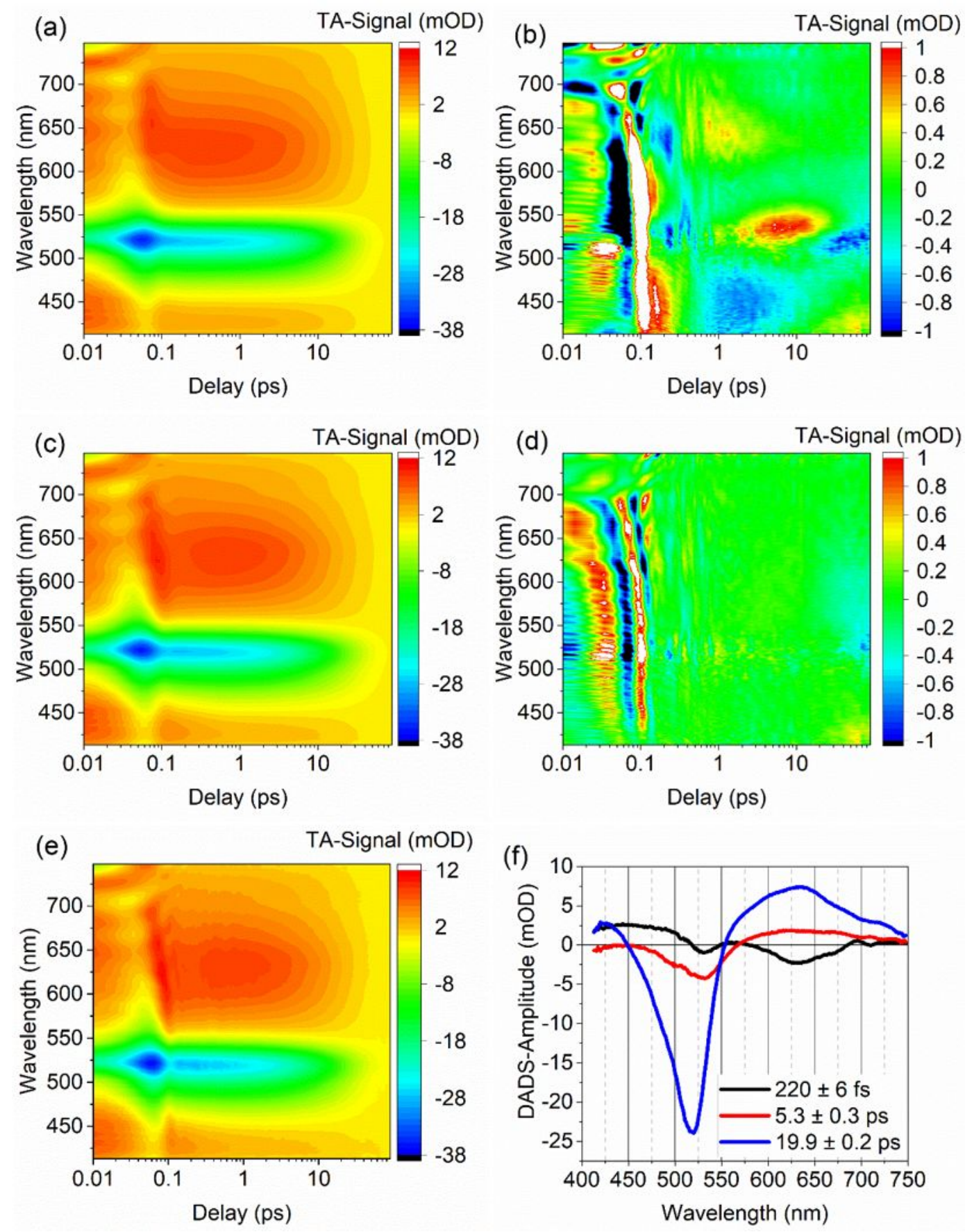

Figure S3: Global Analysis of TA data. (a) Fit and (b) residual from fitting with two exponential decays. (c) Fit, (d) residual and (f) decay-associated difference spectra from fitting with three exponential decays. (e) Original TA data set for reference. 


\section{IVS- and Pump-IVS Data}

Absorption at $\lambda=518 \mathrm{~nm}$ decreases from 1.032 OD to 0.98 OD i.e. by $5 \%$. The comparison of the normalized absorption spectra (Figure S4b) shows no additional changes in the spectrum shape, indicating that the photo-damage does not result in any species absorbing in the observed spectral range.

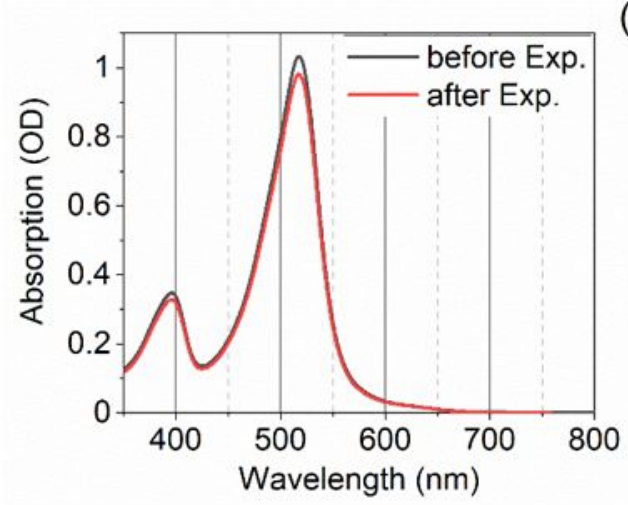

(a)

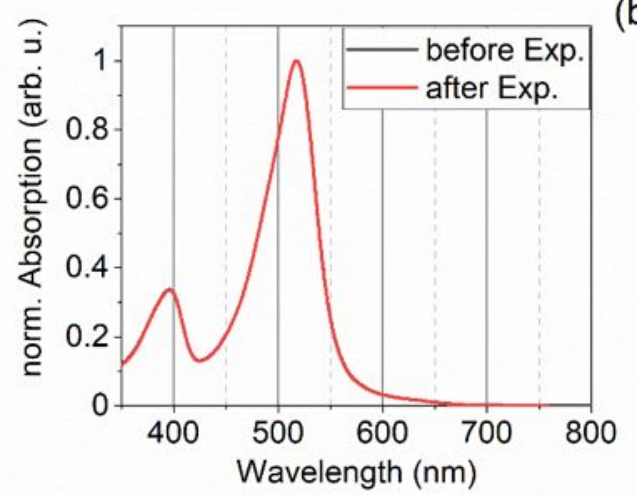

(b)

(c)

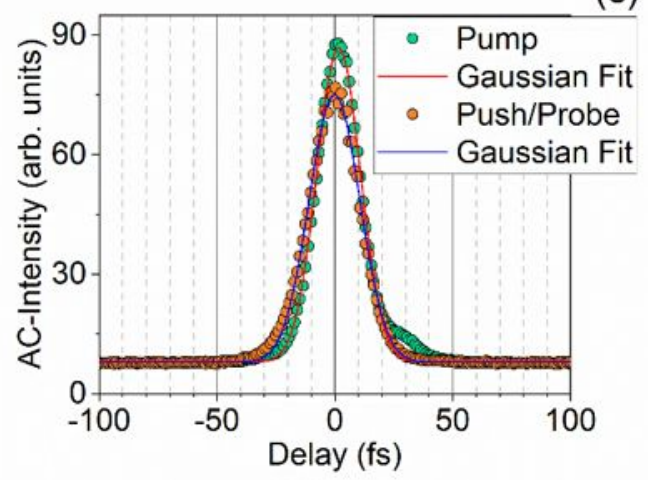

Figure S4: (a) Absolute and (b) normalized ground state absorption before and after IVS- and P-IVS measurements, recorded in a $1 \mathrm{~mm}$ cuvette. (c) Autocorrelation of pump, push and probe pulses.

In order to monitor the signal oscillations induced by the actinic pump pulse thoroughly, a 3 ps delay range was scanned in a single experiment in contrast to the $1 \mathrm{ps}$ scan ranges at selected later delays (Figure S5 a). P-IVS measurement with $\mathrm{T}<2 \mathrm{ps}$ did not yield new information, since the oscillations induced by the actinic pump pulse dominated the signal. The arrival of the push pulse in the P-IVS measurements manifests as a coherent artefact at the indicated T delays.

The Gaussian fit of the autocorrelation (Figure S4c) yields $21.7 \mathrm{fs}$ FWHM for the pump pulse and 25.4 $\mathrm{fs}$ for the push/probe pulse. This corresponds to pulse durations of $15.3 \mathrm{fs}$ and $18.0 \mathrm{fs}$, respectively. 


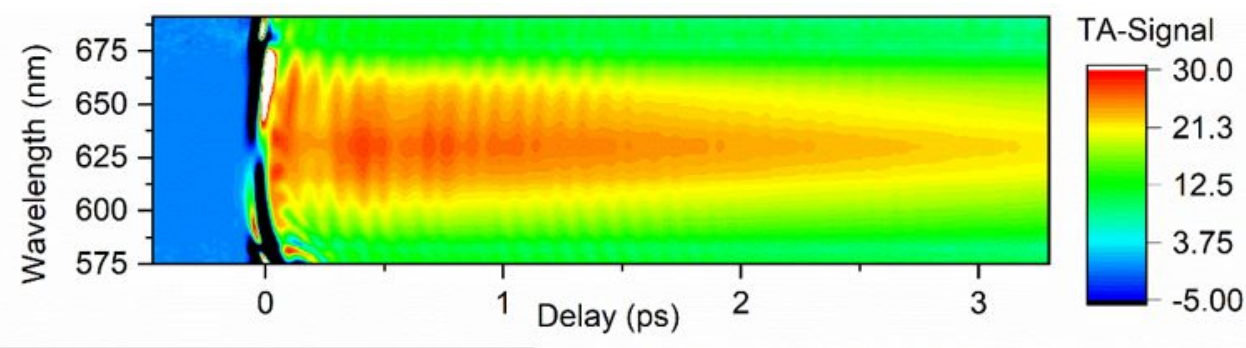

(a)

21.3

12.5

3.75

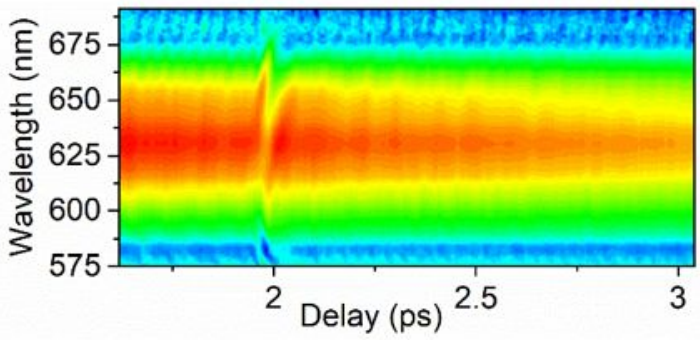

TA-Signal

(b) $\square 25$
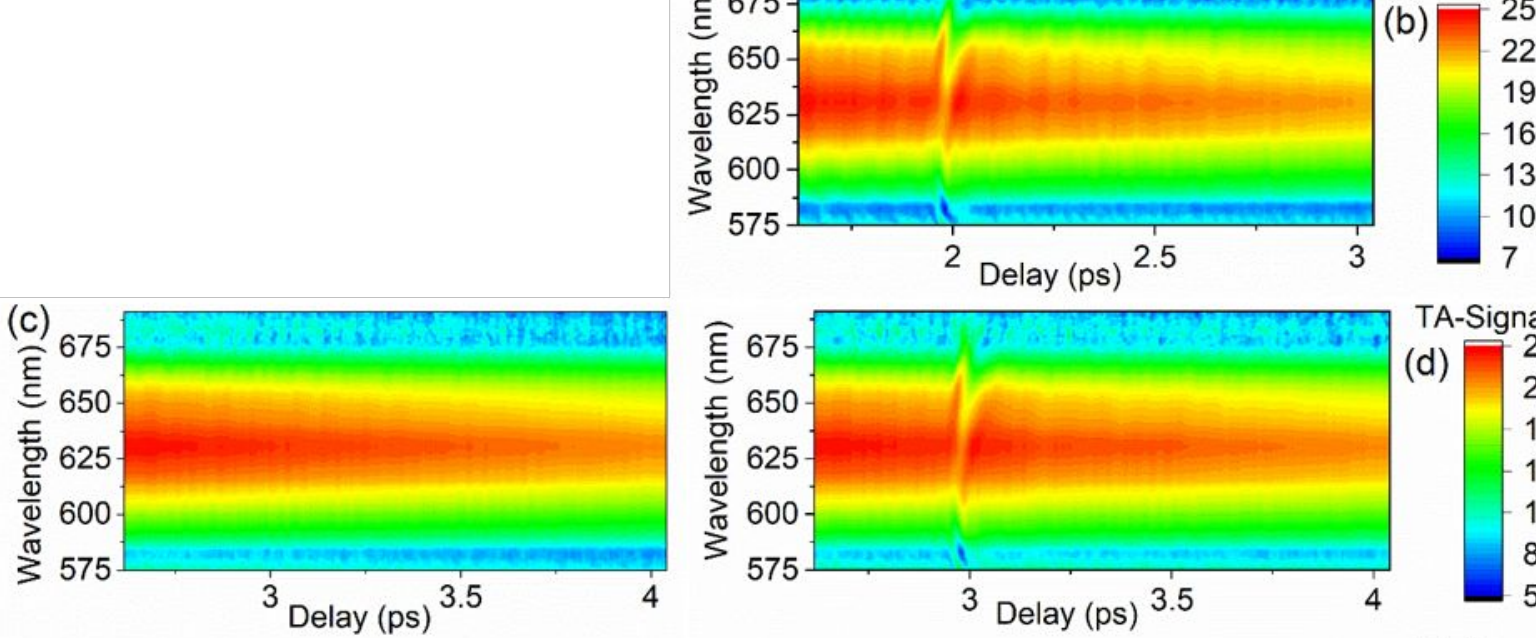

TA-Signal

(d)

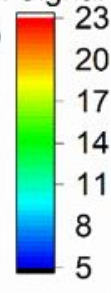

(e) 675

约 650

吉 625

$\frac{\Phi}{\omega} 600$

元 575
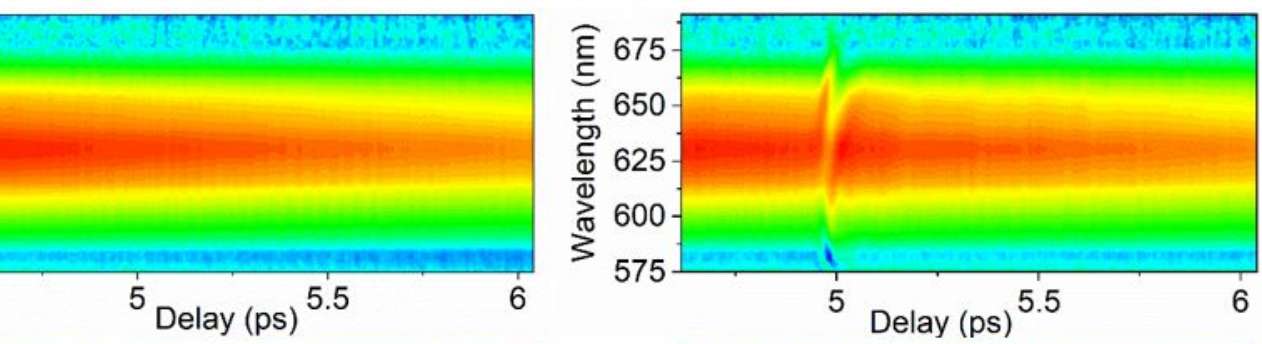

TA-Signal

(f)

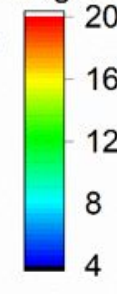

(g) 675

હิ 650

吉 625

ब 600

而 575
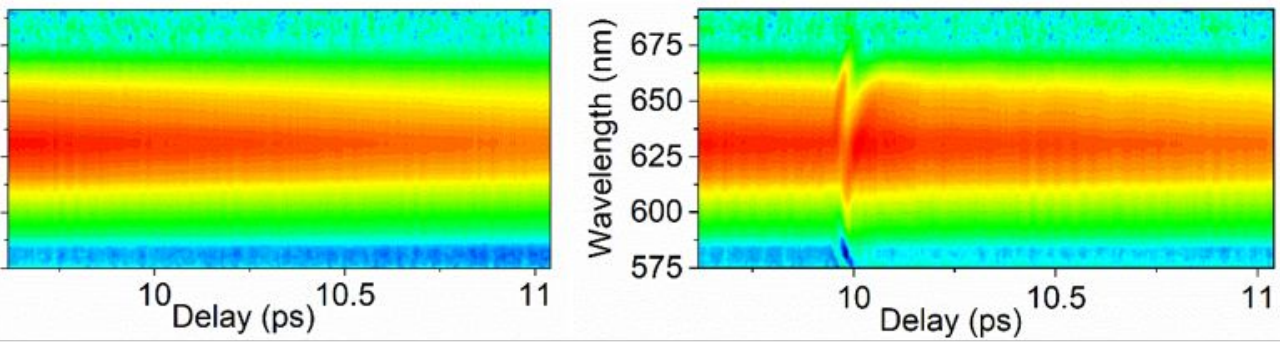

TA-Signal

(h)
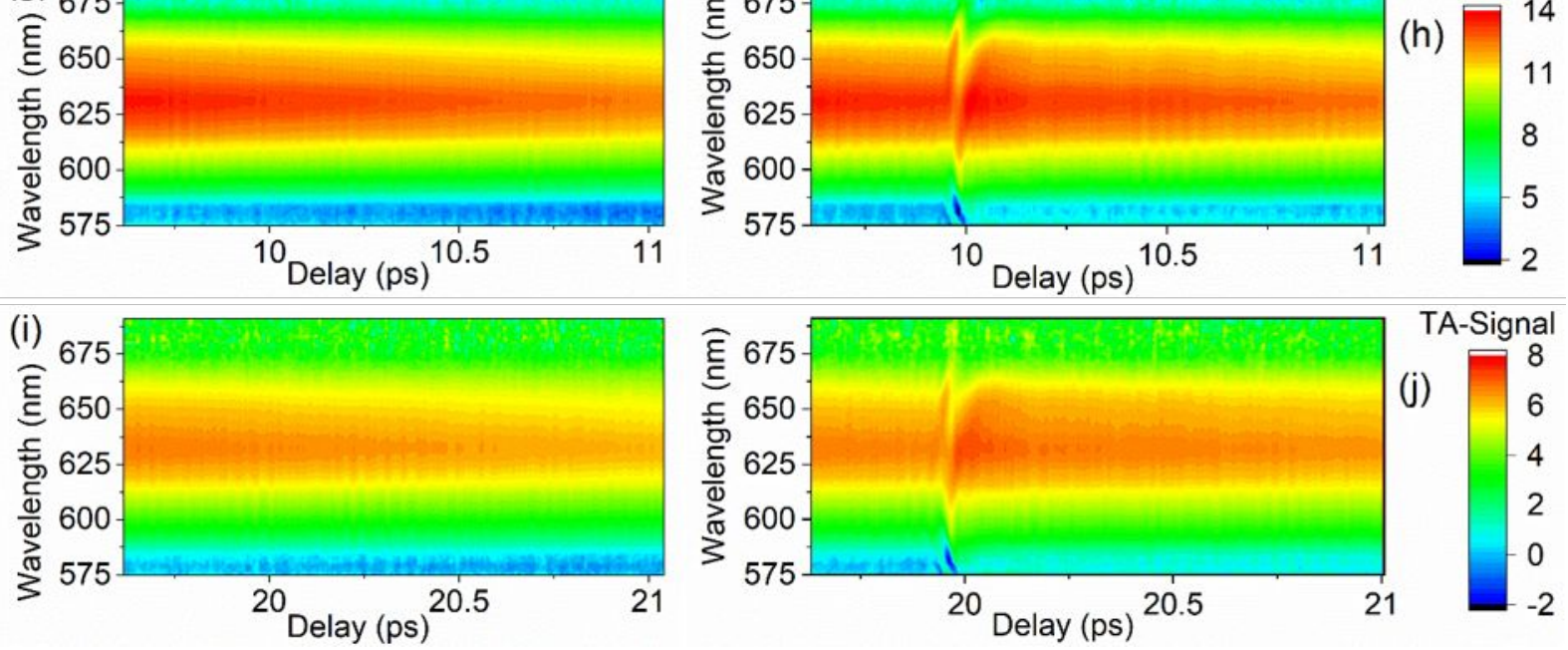

Figure S5: Full IVS and P-IVS data sets (left and right column, respectively). a) IVS T=0 ps, b) P-IVS T=2ps.

c) and d): $T=3 \mathrm{ps}$, e) and f) $T=5 \mathrm{ps}, \mathrm{g}$ ) and h) $T=10 \mathrm{ps}$, i) and j) $T=20 \mathrm{ps}$. 


\section{Biexponential Fitting of IVS- and P-IVS Data}
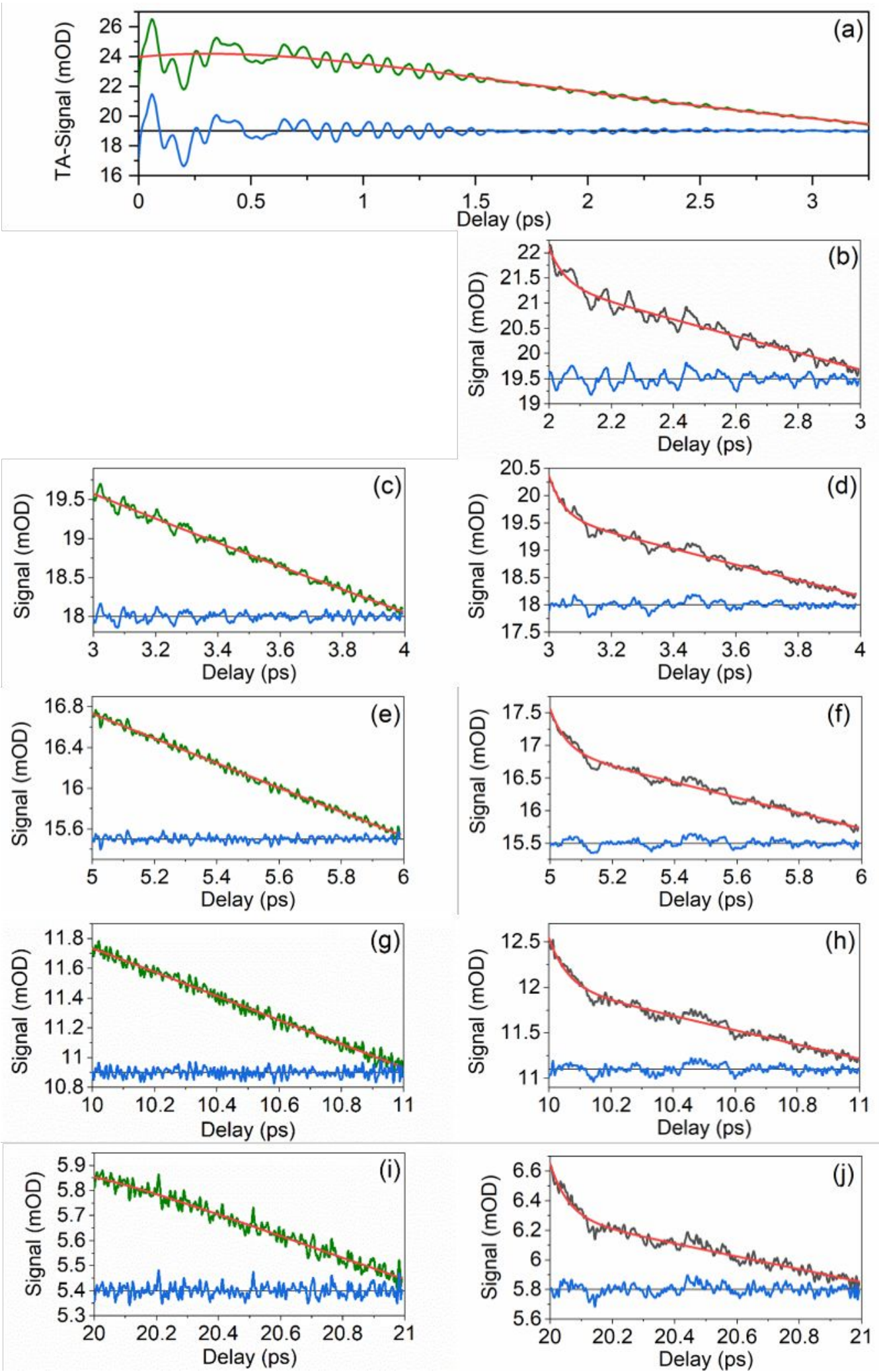
Figure S6: Biexponential fitting of IVS and P-IVS signal a) at $650 \mathrm{~nm}, b-j$ ) average from $647-652 \mathrm{~nm}$. IVS signal (green), P-IVS signal (black), biexponential fit (red) and residual thereof (blue). a) IVS T=0 ps, b) P-IVS $T=2 p s$. c) and d): $T=3$ ps, e) and f) $T=5$ ps, g) and h) $T=10 p s$, i) and j) $T=20$ ps.

The spectra shown in Figure $2 \mathrm{~d}$ are obtained by selecting traces with 1 ps temporal length from the residual shown in Figure $\mathbf{S 6}$ and starting at the indicated delay. This selected trace is then multiplied by a Gaussian window to avoid artefacts in the FFT. The spectra shown in Figure 3d are obtained by Fourier transformation of the residuals shown in Figure $\mathrm{S} 6 \mathrm{~b}$-j. In all cases zero padding was applied.

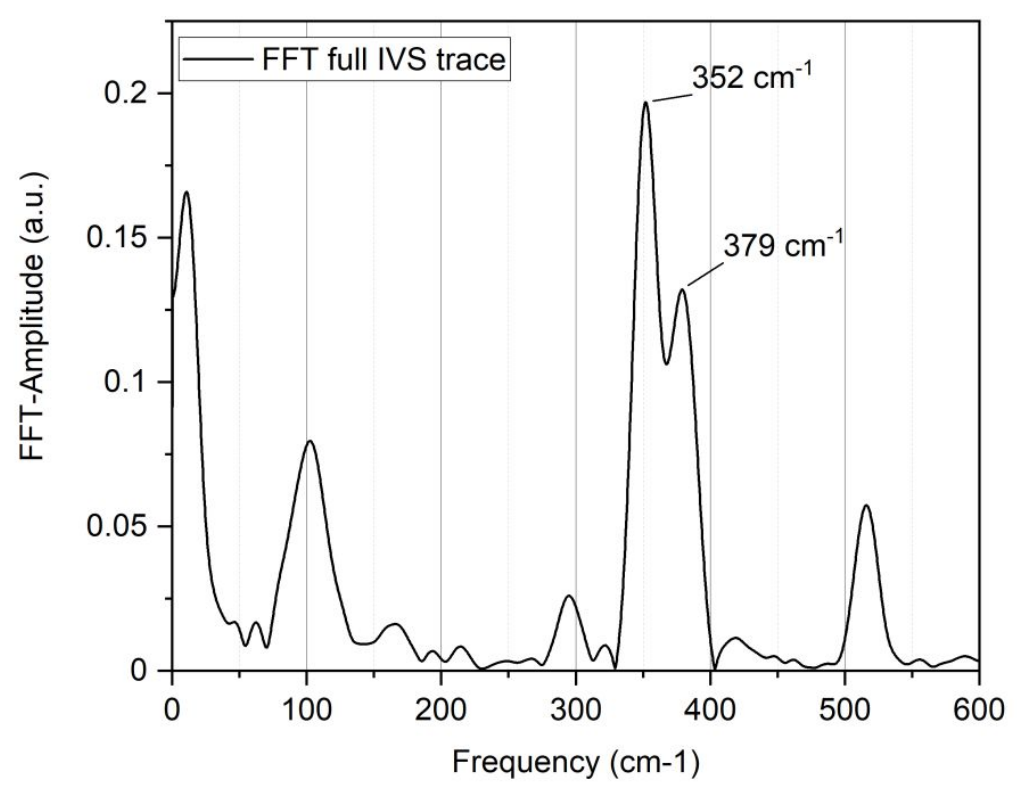

Figure S7: FFT of full 3.2 ps IVS trace. A Gaussian window and zero padding was applied.

The FFT spectrum given in Figure S7 is based on the full recorded IVS-trace shown in Figure S6a. Other than in the SW-FFT (1 ps trace length) the solvent mode $(379 \mathrm{~cm}-1)$ and the ligand deformation mode $(352 \mathrm{~cm}-1)$ can be resolved here. The obtained frequencies are in good agreement with those obtained from the oscillatory fit (Figure 2, Table 1). 


\section{Decay of the $100 \mathrm{~cm}^{-1}$ Mode in Sliding-Window FFT}

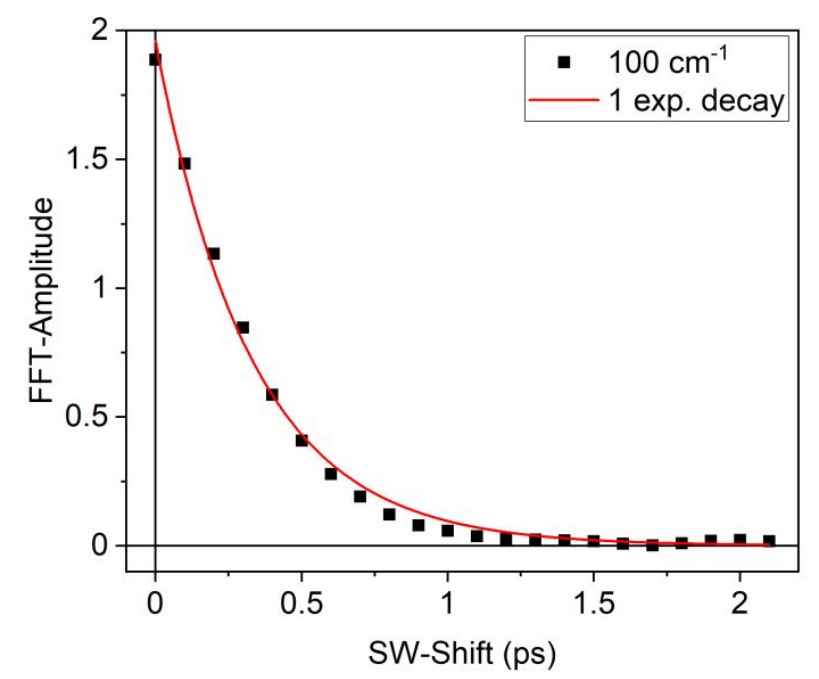

Figure S8: FFT-Amplitude at $\tilde{v}=100 \mathrm{~cm}^{-1}$ from SW-FFT (100 fs step size, 1 ps trace length) (black) and exponential fit: Amplitude: 1.96 arb. u.; Offset: 0 arb. u. (constrained); Lifetime: $331 \pm 12$ fs.

\section{Oscillatory Fitting of IVS and P-IVS data}

The oscillatory fitting of the IVS and P-IVS signal modulations (Figure $2 b$ and $3 b$ ) are calculated as following. The observed signal modulation $S$ is modelled as a sum of damped sine functions $v_{i}$ corresponding to the dephasing wavepacket motions with an individual amplitude $A_{i}$, dephasing time $\tau_{i}$, oscillation period $T_{i}$ and phase $\operatorname{shift} \varphi_{i}$.

$$
\begin{gathered}
S=\sum_{i=1}^{n} v_{i} \\
v_{i}=A_{i} * \exp \left(-\frac{t}{\tau_{i}}\right) * \sin \left(\frac{2 \pi * t}{T_{i}}+\varphi_{i}\right)
\end{gathered}
$$

The fit optimization is done using a Levenberg-Marquardt algorithm as implemented in LabVIEW' 2018. 
7. Calculation of Low-Frequency Vibrations in ${ }^{3} \mathrm{MLCT}$ and ${ }^{3} \mathrm{MC}$

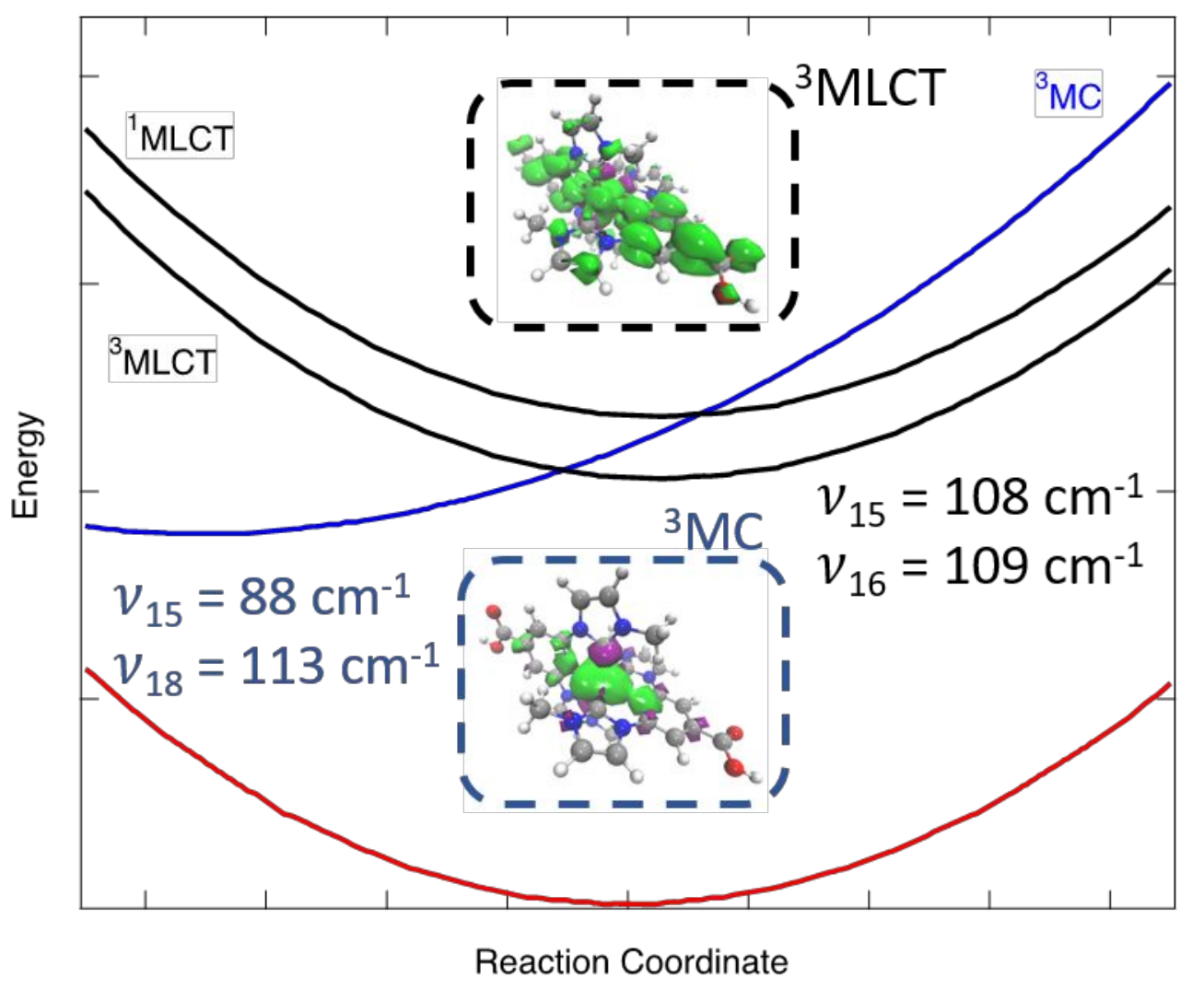

Figure S9: Schematic representation of symmetric Fe-L stretch vibrations within the ${ }^{3} M L C T$ and ${ }^{3} M C$ state. 


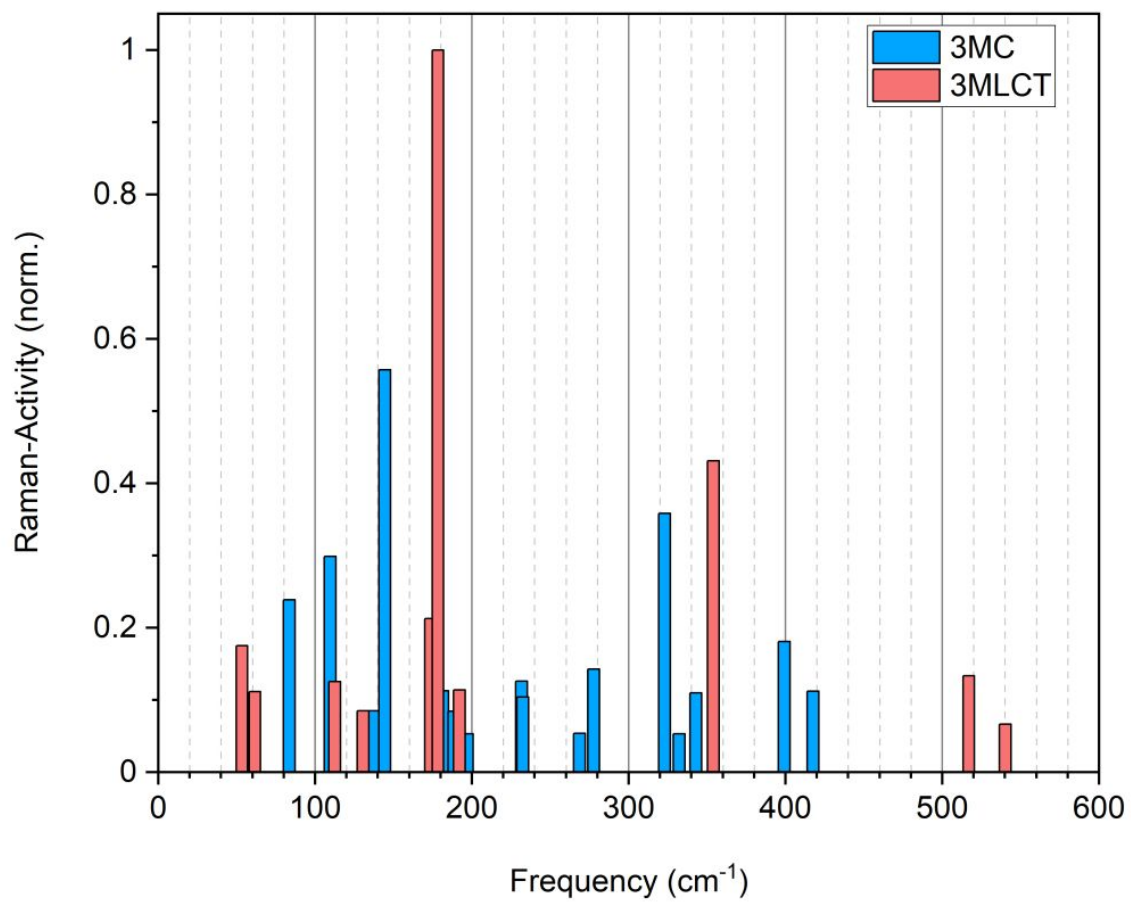

Figure S10: Normalized Raman activities of ${ }^{3} \mathrm{MC}$ and ${ }^{3} \mathrm{MLCT}$ states at frequencies $<600 \mathrm{~cm}^{-1}$. Modes with activities $<0.05$ omitted for clarity. 